\title{
Production and Polarization Effects in Some Tau-Lepton Decays
}

\author{
A. Ilakovac ${ }^{\mathrm{a}}$

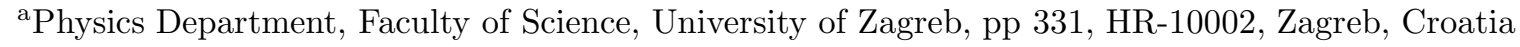

The conditions for the independence of decays of the spin- $1 / 2$ resonances on the production mechanism of the resonances and on polarizations of the incoming and outgoing particles are derived and applied in the case of several tau-lepton decays. The necessity for inclusion of the influence of the production mechanism in the evaluation of the lepton flavour violating decays is stressed.

\section{INTRODUCTION}

The theoretical results for lepton flavour violating (LFV) branching ratios (BR) are usually given in terms of the decay rates, independently of the production mechanism of the tau leptons. In this paper the total cross sections involving decaying resonances are studied. The cross sections are evaluated exactly and assuming the independence of decay processes of the production mechanism. The deviations of approximative cross section from the exact cross section are analyzed. The aim of this work is to show that the exact calculation should be applied to LFV processes, too.

The independence of resonance decay rates of the production mechanism has been conjectured in the early sixties [1, 2, 3, 3, and recently it has been refined for a scalar resonance [4]

$\frac{d \sigma_{A}}{d M^{2} d^{3} P}=\frac{d \sigma_{R}(M)}{d^{3} P} \frac{M \Gamma_{R \rightarrow A}(M)}{\pi \mathcal{D}_{R}(M)}$.

$d \sigma_{A}$ is the cross section of the process $1+2 \rightarrow$ $X+(R \rightarrow A)(1$ and 2 are incoming particles, $R$ is a resonance, $A$ are decay products of $R$, and $X$ are all particles not included in the $R$ decay). $d \sigma_{R}(M)$ is the cross section of the process $1+2 \rightarrow X+R$ (where $R$ is treated as a free particle of an unphysical mass $M) . \Gamma_{R \rightarrow A}(M)$ is the decay rate of $R$ ( $R$ again treated as a free particle of unphysical mass $M)$. $\mathcal{D}_{R}(M)$ is the usual denominator of the resonance- $R$ propagator,

$\mathcal{D}_{R}(M)=\left(M_{0}^{2}-M^{2}\right)^{2}+M_{0}^{2} \Gamma_{R}^{2}(M)$
( $M_{0}$ is the physical mass of the resonance $R, \Gamma_{R}$ is its total decay rate). $P$ is the four-momentum of the resonance $R$ and $M^{2}=P^{2}$. Equation (1) is valid for one decaying resonance in the amplitude. It suggests that the process $1+2 \rightarrow X+(R \rightarrow A)$ can be viewed as a sequence of two independent processes, $1+2 \rightarrow X+R$ and $R \rightarrow A$. Therefore, the decay rate of the resonance $R$ is independent of the production mechanism. The approximate integral form of Eq. (11), valid for narrow resonances, which follows from the approximate relation

$\int_{0}^{\infty} M \mathcal{D}_{R}(M)^{-2} d M^{2}=\frac{\pi}{\Gamma_{R}}$,

is given by

$\sigma_{A}=\sigma_{R} \frac{\Gamma_{R \rightarrow A}}{\Gamma_{R}}$.

Equation (11) can be generalized for several decaying resonances in the amplitude, and this generalization is especially simple for narrow resonances.

For a scalar resonance, the product form for the total cross section $(\sigma \mathrm{PF})$ (1) is a consequence of the product form of the corresponding matrix element,

$\mathcal{M}_{A}=\mathcal{M}_{R} i \mathcal{D}_{R}^{-1} \mathcal{M}_{R \rightarrow A}$,

with notation as in (1). $\mathcal{M}_{R}$ and $\mathcal{M}_{R \rightarrow A}$ are the same matrix elements that appear in the evaluation of the cross section $1+2 \rightarrow R+X$ and $R \rightarrow A$.

In the case of spin- $1 / 2$ and vector resonances or resonances with more complicated spin-tensor 
structure, the product form for matrix elements (5) is not so simple and the product form for the differential cross section (ii) is not generally valid. The product form for the differential cross section (1) for a vector resonance in the amplitude has been studied in [4. The conditions for the product formula for the differential cross section or total cross section for the processes with one and two spinor resonances are given here.

\section{CONDITIONS FOR $\sigma$ PF FOR ONE SPIN- $1 / 2$ RESONANCE}

For a spin- $1 / 2$ resonance, the matrix elements $\mathcal{M}_{R \rightarrow A}, \mathcal{M}_{R}$ and $\mathcal{M}_{A}$ read

$$
\begin{aligned}
\mathcal{M}_{R \rightarrow A} & =\bar{U}_{A} u_{R}, \\
\mathcal{M}_{R} & =\bar{u}_{R} U_{S}, \\
\mathcal{M}_{A} & =\mathcal{D}_{R}^{-1} \bar{U}_{A} \mathcal{P}_{R} U_{S},
\end{aligned}
$$

where $u_{R}$ is the wave function of the resonance, $U_{A}$ and $U_{S}$ ("spinor amplitudes") are general spinor functions describing the decay products of the the resonance $(A)$ and scattering process $1+2 \rightarrow X+R$ (without the resonance wave function) and $\mathcal{P}_{R}=\mathbb{P}+M_{0}$ is the numerator of the propagator of the resonance. Spinor functions $U_{A}$ and $U_{S}$ contain all information on momenta and polarizations of the decay products $\{A\}$ and particles $1,2,\{X\}$ respectively. The corresponding decay rates and differential cross sections read

$$
\begin{aligned}
\Gamma_{R \rightarrow A} & =\left(16 M \pi^{2}\right)^{-1} \operatorname{Tr}\left[\mathcal{P}_{R} T\right], \\
E \frac{d \sigma_{R}}{d^{3} P} & =\frac{\pi}{4 F} \operatorname{Tr}\left[\mathcal{P}_{R} S\right], \\
E \frac{d \sigma_{A}}{d^{3} P d M^{2}} & =\frac{\pi}{32 F} \operatorname{Tr}\left[S \mathcal{P}_{R} T \mathcal{P}_{R}\right],
\end{aligned}
$$

where $E=\left(M^{2}+\mathbf{P}^{2}\right)^{\frac{1}{2}}$ is the energy of the resonance, $F=E_{1} E_{2}\left|\mathbf{v}_{1}-\mathbf{v}_{2}\right|$, and

$$
\begin{aligned}
T & =\sum_{\left\{\lambda_{A}\right\}} \int d \operatorname{LIPS}_{A} U_{A} \times \bar{U}_{A}, \\
S & =\sum_{\left\{\lambda_{X}\right\}} \int d \phi_{X} U_{S} \times \bar{U}_{S},
\end{aligned}
$$

with $\left\{\lambda_{A}\right\},\left\{\lambda_{X}\right\}$ and $d \operatorname{LIPS}_{A}$ and $d \phi_{X}$ representing the polarizations of $\{A\}$ and $\{X\}$ particles, Lorentz invariant phase space of particles
$\{A\}$ and phase space of $\{X\}$ particles, respectively.

The most general form of $T$ is

$T=T_{1}+T_{2}+T_{3}^{\mu \nu} \sigma_{\mu \nu}+\mathbb{T} \gamma_{5}+T_{5} \gamma_{5}$.

The product form for the differential cross section is satisfied if the product of the traces from Eqs. (7) and (8) is equal to the trace from Eq. (9). The sufficient condition for that equality to be satisfied is

$\mathcal{P}_{R} T \mathcal{P}_{R}=\mathcal{P}_{R} \frac{1}{2} \operatorname{Tr}\left[T \mathcal{P}_{R}\right]$,

with the most general solution for $T$,

$T=T_{1}+T_{2}+T_{4} \bar{P}+T_{5} \gamma_{5}$,

$\left(\bar{P}=P / M_{0}\right)$. Although the condition 1 13) is not the only one that realizes the product form for the differential cross section (13) for one resonance in the scattering process $1+2 \rightarrow R+X$, it is the only one that remains for more than one decaying resonance in the scattering process.

If the summation over all polarizations of $\{A\}$ particles and integration over complete $d \operatorname{LIPS}_{A}$ phase space is performed, $T$ depends only on the momentum of the resonance $P$, and its most general form,

$T=T_{1}+T_{2} \bar{P}+T_{4} \bar{P}+T_{5} \gamma_{5}$

(form factors $T_{i}$ depend only on $P^{2}$ ) satisfies the product form condition (13). Therefore, if an experiment is performed with a $4 \pi$ detector, and polarizations are not measured, the $\sigma \mathrm{PF}$ is satisfied for processes with one decaying spin- $1 / 2$ resonance. The result can be generalized for processes with any number of decaying spin- $1 / 2$ resonances.

In the following, three possible situations which deviate from the situation described by Eq. (15), will be discussed,

1. polarization of the decay products $\{A\}$ of the resonance,

2. a process with two or more spin- $1 / 2$ resonances,

3. partial integration over phase space for processes with two spin- $1 / 2$ resonances. 


\section{3. $\sigma$ PF AND POLARIZATION}

The description of the polarization of massless spin- $1 / 2$ particles, massive spin- $1 / 2$ and vector particles and massless vector particles is different. Helicity of the massless spin- $1 / 2$ particles is completely determined by the $V \pm A$ vertices and no additional four-vector is needed for the description of the particle polarization. For massive spin- $1 / 2$ and vector particles one has to introduce additional four-vector along with the particle four-momentum for a complete description of the particle state. For instance, for a massive spin- $1 / 2$ particle of mass $m$ and four-momentum $p$, the four vectors describing the particle state are,

$p=(E, p \hat{\mathbf{p}}), s=(p, E \hat{\mathbf{p}}) / m$.

( $\hat{\mathbf{p}}$ is the unit vector in the direction of $\mathbf{p}$ ). The direction of the spacial part of the four-vectors $p$ and $s$ are the same. That follows from the definition of the helicity quantization axis and the fact that the little group of the massive particle is attached to its rest frame. For massless vector particles, the additional four-vector that is necessary for the description of a helicity of the state, $\eta$, is independent of the four-momentum of the particle, and it represents the laboratory frame in which the quantization of the masslessvector-particle spin is performed. In the laboratory frame it is a pure time-like four-vector.

If among the polarized particles of the decay products of the resonance $R$ only spin- $1 / 2$ and massive vector particles appear, the complete integration over $d \operatorname{LIPS}_{A}$ gives $T$ again the structure (15) that assures $\sigma \mathrm{PF}$. The presence of polarized massless vector particles among the decay products of the resonance $R$ leads to additional terms of the $T$ even after the complete integration over $d \operatorname{LIPS}_{A}$,

$$
\begin{aligned}
T & =T_{1}+T_{2 A} \overline{\bar{P}}+T_{2 B} \not+T_{3} \sigma_{\mu \nu} \eta^{\mu} \bar{P}^{\nu} \\
& +T_{4 A} \overline{\bar{P}}+T_{4 B} \not \gamma_{5}+T_{5} \gamma_{5},
\end{aligned}
$$

which cannot be removed by the most general gauge transformation of polarization vectors of the type, $\varepsilon\left(p, \lambda_{i}\right) \rightarrow \varepsilon\left(p, \lambda_{i}\right)+\alpha_{i} p$, where $p$ is the four-momentum of the massless vector particle and $\alpha_{i}$ are constants. The fourth and sixth term in Eq. (17) violate the product form condition (13).

\section{4. $\sigma$ PF FOR TWO RESONANCE PRO- CESSES}

The expected $\sigma \mathrm{PF}$ equation for a two narrowresonance process is

$\sigma_{A B}=\sigma_{R_{1} R_{2}} \frac{\Gamma_{R_{1} \rightarrow A}}{\Gamma_{R_{1}}} \frac{\Gamma_{R_{2} \rightarrow B}}{\Gamma_{R_{2}}} \equiv \sigma_{A B}^{P F}$

$\left(\sigma_{A B} \equiv \sigma_{1+2 \rightarrow\left(R_{1} \rightarrow A\right)\left(R_{2} \rightarrow B\right)}, \quad \sigma_{R_{1} R_{2}} \equiv\right.$ $\left.\sigma_{1+2 \rightarrow R_{1} R_{2}}\right)$. The relevant matrix elements for studying $\sigma \mathrm{PF}$ are

$$
\begin{aligned}
\mathcal{M}_{A} & \equiv \mathcal{M}_{R_{1} \rightarrow A}=\bar{U}^{A} u^{R_{1}}, \\
\mathcal{M}_{B} & \equiv \mathcal{M}_{R_{2} \rightarrow B}=\bar{u}^{R_{2}} U^{B}, \\
\mathcal{M}_{R_{1} R_{2}} & \equiv \mathcal{M}_{1+2 \rightarrow R_{1} R_{2}}=\bar{u}^{R_{1}} s u^{R_{2}}, \\
\mathcal{M}_{A B} & =\bar{U}^{A} \mathcal{P}^{R_{1}} s \mathcal{P}^{R_{2}} U^{B} \mathcal{D}_{R_{1}}^{-1} \mathcal{D}_{R_{2}}^{-1}
\end{aligned}
$$

( $u^{R_{1}}$ and $u^{R_{2}}$ are the wave functions of the resonances, $U^{A}$ and $U^{B}$ describe the resonance products, $\mathcal{P}^{R_{1}}$ and $\mathcal{P}^{R_{2}}$ are the numerators of the propagators of the resonances, $\mathcal{D}_{R_{1}}$ and $\mathcal{D}_{R_{2}}$ are the corresponding denominators of the propagators, and $s$ is $1+2 \rightarrow R_{1} R_{2}$ amplitude without the resonance wave functions). Squaring the amplitude, using the usual trick for the subdivision of the phase space of outgoing particles ( 4,5$])$,

$$
\begin{aligned}
d L I P S_{A B}= & \frac{1}{(2 \pi)^{2}} d M_{R_{1}}^{2} d M_{R_{2}}^{2} d L I P S_{R_{1} R_{2}} \\
& d L I P S_{A} d L I P S_{B},
\end{aligned}
$$

integrating out the delta functions, and integrating over the squares of unphysical resonance masses, one obtains the approximative expression (valid for narrow resonances) for the total cross section (LHS of Eq. (18))

$$
\begin{gathered}
\sigma_{A B} \approx \frac{P \lambda^{-\frac{1}{2}} F^{-1}}{2^{8} \pi^{2} \Gamma_{R_{1}} \Gamma_{R_{2}} M_{R_{1}}^{0} M_{R_{2}}^{0}} \int d \Omega_{R_{1}} d L_{A} d L_{B} \\
\sum_{i j} S_{i j}^{I J} \operatorname{Tr}\left[\mathcal{P}^{R_{1}} t^{A} \mathcal{P}^{R_{1}} \tilde{\gamma}_{i I} \mathcal{P}^{R_{2}} t^{B} \mathcal{P}^{R_{2}} \tilde{\gamma}_{j J}\right] \\
=\frac{P \lambda^{-\frac{1}{2}} F^{-1}}{2^{6} \pi^{2} \Gamma_{R_{1}} \Gamma_{R_{2}}} \int d \Omega_{R_{1}} \\
\sum_{i j} S_{i j}^{I J} \operatorname{Tr}\left[\mathcal{P}^{R_{1}} \tilde{\Gamma}_{A} \mathcal{P}^{R_{1}} \tilde{\gamma}_{i I} \mathcal{P}^{R_{2}} \tilde{\Gamma}^{B} \mathcal{P}^{R_{2}} \tilde{\gamma}_{j J}\right]
\end{gathered}
$$


$\left(\lambda=\left(p_{1}+p_{2}\right)^{2}, P=\left|\mathbf{P}_{R_{1}}\right|=\left|\mathbf{P}_{R_{2}}\right|, M_{R_{1}}^{0}\right.$ and $M_{R_{2}}^{0}$ are physical masses of the resonances; $d \Omega_{R_{1}}$ is differential solid angle of $\hat{\mathbf{P}}_{R_{1}}, d L_{A}$ and $d L_{B}$ are remnants of $d \operatorname{LIPS}_{A, B}$ phase spaces after integration over delta functions; $\mathcal{P}^{R_{a}}=\mathbb{P}_{R_{a}} \pm M_{R_{a}}$, $a=1,2$, are numerators of $R_{a}$ propagators - plus sign is for a particle resonance and minus sign for an antiparticle resonance;

$t^{A}=\gamma_{0} U_{A}^{\dagger} \times U_{A}$,

$t^{B}=\gamma_{0} U_{B}^{\dagger} \times U_{B}$,

are absolute squares of the spinor amplitude decay products of resonances $R_{1,2}$,

$\sum_{i j} S_{i j}^{I J} \tilde{\gamma}_{i I} \tilde{\gamma}_{j J} \equiv S=\gamma_{0} s^{\dagger} \gamma_{0} \times s$

$\tilde{\gamma}_{i}$ are Dirac algebra matrices and $I$ is a set of Lorentz indices assigned to a particular $\tilde{\gamma}_{i}$ matrix; $\sum_{i j} S_{i j}^{I J}$ are tensor functions which are part of the absolute square of the spinor amplitude $s$; the quantities

$\tilde{\Gamma}_{A, B}=\int d L_{A, B} t^{A, B}$

are "spinor decay rates" of resonances $R_{1,2}$ because $\left.\Gamma_{R_{1,2} \rightarrow A, B}=\frac{1}{2 M_{R_{1,2}}} \operatorname{Tr}\left[\mathcal{P}^{R_{1}, R_{2}} \tilde{\Gamma}_{A, B}\right]\right)$. The expression for the RHS of Eq. (18) is obtained from (24) by replacement

$$
\begin{aligned}
\sum_{i j} & S_{i j}^{I J} \operatorname{Tr}\left[\mathcal{P}^{R_{1}} t^{A} \mathcal{P}^{R_{1}} \tilde{\gamma}_{i I} \mathcal{P}^{R_{2}} t^{B} \mathcal{P}^{R_{2}} \tilde{\gamma}_{j J}\right] \rightarrow \\
& \sum_{i j} S_{i j}^{I J} \operatorname{Tr}\left[\mathcal{P}^{R_{1}} \tilde{\gamma}_{i I} \mathcal{P}^{R_{2}} \tilde{\gamma}_{j J}\right] \\
& \times \operatorname{Tr}\left[t^{A} \mathcal{P}^{R_{1}}\right] \operatorname{Tr}\left[t^{B} \mathcal{P}^{R_{2}}\right] .
\end{aligned}
$$

The $\sigma \mathrm{PF}$ equation leads to the equality of LHS and RHS of Eq. (28). It can be shown that the only way to satisfy that equality is that the following two conditions are satisfied,

$$
\begin{aligned}
\left(\mathcal{P}^{R_{1}} T^{A} \mathcal{P}^{R_{1}}\right) & =\mathcal{P}^{R_{1}} \frac{1}{2} \operatorname{Tr}\left(T^{A} \mathcal{P}^{R_{1}}\right), \\
\left(\mathcal{P}^{R_{2}} T^{B} \mathcal{P}^{R_{2}}\right) & =\mathcal{P}^{R_{2}} \frac{1}{2} \operatorname{Tr}\left(T^{B} \mathcal{P}^{R_{2}}\right)
\end{aligned}
$$

$\left(T^{A, B}=t^{A, B}\right.$ or $\left.T^{A, B}=\int d L_{A, B} t^{A, B}\right)$. Eqs. (29) are identical in form to Eq. (13), and their most general solutions have the same form as (14),

$$
\begin{aligned}
T^{A} & =T_{1}^{A}+\mathbb{T}_{2}^{A}+T_{4}^{A} P_{4}^{A} \gamma_{5}+T_{5}^{A} \gamma_{5}, \\
T^{B} & =T_{1}^{B}+\mathbb{T}_{2}^{B}+T_{4}^{B} P_{4}^{B} \gamma_{5}+T_{5}^{B} \gamma_{5} .
\end{aligned}
$$

The generalization of $\sigma \mathrm{PF}$ conditions for more than two spin- $1 / 2$ resonances is obvious.

\section{ANALYSIS OF $\sigma$ PF for $e^{-} e^{+} \rightarrow\left(\tau^{-} \rightarrow\right.$ $A)\left(\tau^{+} \rightarrow B\right)$ PROCESSES}

From the above analysis follows that to prove the some process containing only spin- $1 / 2$ resonances satisfies $\sigma \mathrm{PF}$ one has to show that all absolute squares of the spinor amplitudes of spin$1 / 2$ resonances included in the process, or any phase space integral (partial or complete) of these quantities must satisfy the condition (13). That rule will be used to study several $e^{-} e^{+} \rightarrow\left(\tau^{-} \rightarrow\right.$ $A)\left(\tau^{-} \rightarrow B\right)$ ptocesses with respect to the condition (13). Three processes are chosen for the analysis, $e^{-} e^{+} \rightarrow\left(\tau^{-} \rightarrow \pi^{-} \nu_{\tau}\right)\left(\tau^{+} \rightarrow \pi^{+} \bar{\nu}_{\tau}\right)$, $e^{-} e^{+} \rightarrow\left(\tau^{-} \rightarrow l^{-} \bar{\nu}_{l} \nu_{\tau}\right)\left(\tau^{+} \rightarrow l^{+} \nu_{l} \bar{\nu}_{\tau}\right)$ and $e^{-} e^{+} \rightarrow\left(\tau^{-} \rightarrow \pi^{-} \nu_{\tau}\right)\left(\tau^{+} \rightarrow l^{+} \gamma\right)$. First two are Standard Model processes, while the third belongs to the physics beyond the Standard Model. The first one contains one observable scalar per decaying resonance, the second contains one observable lepton per resonance, and the third comprises one resonance as in the first case while the other resonance decays into observable lepton and photon. The parts of the phase space of the unobservable particles can be integrated. In the following analysis simple model of LFV - Standard model with additional heavy neutrinos $[6]$ ) is used.

The spinor decay rates involved in the processes read (complete phase space integration and summation over all polarizations is assumed):

$$
\begin{aligned}
\tilde{\Gamma}_{\tau \rightarrow \pi \nu_{\tau}}= & \bar{G}_{F}^{2} \bar{f}_{\pi}^{2} \frac{\left|V_{u d}\right|^{2}}{2^{3} \pi}\left(1-\frac{m_{\pi}^{2}}{m_{\tau}^{2}}\right) \bar{P}_{\tau} P_{L}, \\
\tilde{\Gamma}_{\tau \rightarrow l^{-} \bar{\nu}_{l} \nu_{\tau}}= & \frac{\bar{G}_{F}^{2}}{3 \times 2^{6} \pi^{3}} \bar{P}_{\tau} P_{L}, \\
\tilde{\Gamma}_{\tau \rightarrow l^{-} \gamma}= & \frac{\alpha_{W}^{3} s_{W}^{2}}{2^{8} \pi^{2} \bar{M}_{W}^{4}}\left(1-\bar{m}_{l}^{2}\right)^{3} \\
& \bar{P}_{\tau}\left(P_{R}+\bar{m}_{l}^{2} P_{L}\right) F_{L F V}^{2} .
\end{aligned}
$$


Barred quantities are dimensionless. They are obtained from the corresponding dimensionfull quantities multiplying them by a power of the tau mass. $G_{F}$ is Fermi constant, $\alpha_{W}=g^{2} /(4 \pi)$; $F_{L F V}=\left(\sum_{i} B_{\tau i}^{*} B_{l i} G\left(\lambda_{i}\right)\right)^{2}$ is a factor which contains information on the magnitude of LFV - see Refs. [6]. Obviously, all spinor decay rates satisfy the $\sigma \mathrm{PF}$ condition (13). Notice the similarity in the structure of the spin dependent parts of all three $\tilde{\Gamma}$-s.

The approximative expressions (the numerator of the $W$-boson propagator s replaced by $M_{W}^{2}$ ) for the absolute squares of spinor amplitudes for these three processes read

$$
\begin{aligned}
t_{\tau \rightarrow \nu_{\tau} \pi} \approx & m_{\tau} \frac{g^{4}}{8} \frac{\bar{f}_{\pi}^{2}}{\bar{M}_{W}^{4}} V_{u d}^{2}\left(\bar{p}_{\pi}-\bar{m}_{\pi}^{2} \bar{P}_{\tau}\right) \\
t_{\tau \rightarrow \nu_{\tau} \nu_{l} l} \approx & m_{\tau}^{-1} g^{4} \bar{M}_{W}^{-4}\left(1-\bar{s}_{\tau l}-\bar{s}_{\nu_{l} l}\right) \bar{p}_{\nu_{l}} P_{L} \\
t_{\tau \rightarrow l \gamma}= & m_{\tau} \frac{s_{W}^{2}\left(1-\bar{m}_{l}^{2}\right.}{2^{9} \pi^{4} \bar{M}_{W}^{4}} F_{L F V}^{2} \\
& \bar{p}_{\gamma}\left(P_{L}+\bar{m}_{l}^{2} P_{R}\right)
\end{aligned}
$$

$\left(\bar{s}_{\tau l}=\left(\bar{p}_{\tau}-\bar{p}_{l}\right)^{2}, \bar{s}_{\nu_{l} l}=\left(\bar{p}_{\nu}+\bar{p}_{l}\right)^{2}\right)$, The structures of the spin dependent parts of all three $t$-s are similar. Further, none of three absolute squares of spinor amplitudes satisfies the $\sigma \mathrm{PF}$ condition (13). Therefore, a deviation from the $\sigma \mathrm{PF}$ is expected if the complete integration over phase space is not peformed. The phase space measures of all three processes contain the differentials of solid angles of the two observable particles. For the process $e^{-} e^{+} \rightarrow\left(\tau^{-} \rightarrow l^{-} \bar{\nu}_{l} \nu_{\tau}\right)\left(\tau^{+} \rightarrow\right.$ $\left.l^{+} \nu_{l} \bar{\nu}_{\tau}\right)$, we performed the following integrations over phase space. We defined the difference of the differentials of the RHS and LHS of Eq. (18)

$d \Delta \sigma=d \sigma_{A, B}-\frac{1}{\Gamma_{\tau}^{2}} d \sigma_{R_{1} R_{2}} d \Gamma_{A} d \Gamma_{B}$.

Next, the unit spheres of the solid angle variables of the measurable particles were subdivided into two hemispheres (left $L$ and right $R$ with respect to the direction of the incoming electron). So four sectors with respect to the solid angle integrations of observable particles were obtained $(L L, L R, R L$ and $R R)$. Further, we performed a complete integration over one of the four parts of the phase space and at the same time over the remaining part of the phase space. The results evaluated for center of mass energy $\lambda^{1 / 2}=\sqrt{20} m_{\tau}$ are given in the following table.

\section{Table 1.}

The deviation of the cross section from the product form

\begin{tabular}{lr}
\hline sector & \multicolumn{1}{c}{$\Delta \sigma\left(\mathrm{GeV}^{-2}\right)$} \\
\hline$L L$ & $-7.67 \times 10^{-10}$ \\
$L R$ & $7.44 \times 10^{-10}$ \\
$R L$ & $7.82 \times 10^{-10}$ \\
$R R$ & $-7.47 \times 10^{-10}$ \\
$L L+L R+R L+R R$ & $0.11 \times 10^{-10}$ \\
\hline
\end{tabular}

The integration is ten dimensional. The integration was performed by a simple, non-adaptive Monte-Carlo program. Therefore, the result obtained for the sum of the four contributions can be considered as consistent with zero (it tends to zero as one enlarges the number of integration points). On the other side, the cross sections for the total decay rate for $e^{-} e^{+} \rightarrow\left(\tau^{-} \rightarrow\right.$ $\left.l^{-} \bar{\nu}_{l} \nu_{\tau}\right)\left(\tau^{+} \rightarrow l^{+} \nu_{l} \bar{\nu}_{\tau}\right)$, evaluated using the product form formula (LHS of Eq. (18)), is

$\sigma^{P F}=4.42 \times 10^{-9} \mathrm{GeV}$.

The relative deviation of from the $\sigma \mathrm{PF}$ is of the order of $20 \%$. The similarity of the structures of the spin dependent parts of all three $t$-s indicates that the relative deviation of from the $\sigma \mathrm{PF}$ for other two processes is of the same order of magnitude.

In the literature, LFV decay rates are usually theoretically evaluated assuming the $\sigma \mathrm{PF}$. On the other side, measurements of LFV processes are performed by detectors which are not completely $4 \pi$ detectors. Therefore, in principle, one should include the dependence of the LFV decay rates on the production mechanism when presenting the theoretical results.

\section{CONCLUSION}

The conditions for the product form of a decay rate $(\sigma \mathrm{PF})$ of an amplitude containing one or more spin- $1 / 2$ resonances have been found. The analysis of the $\sigma \mathrm{PF}$ has been performed for one 
and two resonances, for unpolarized particles and complete integration over phase space of outgoing particles, for polarized particles and complete integration over phase space of outgoing particles, and for unpolarized particles and partial integration over phase space. It has been found that the $\sigma \mathrm{PF}$ is not satisfied if decay products of the resonance contain polarized massless spin- 1 particle(s) even for complete integration over phase space or if partial integration over phase space is performed, regardless of the polarization of the particles. The formalism has been applied to three $e^{-} e^{+} \rightarrow\left(\tau^{-} \rightarrow A\right)\left(\tau^{+} \rightarrow B\right)$ processes, two Standard Model processes and one lepton flavour violating (LFV) process. These three examples show explicitely that complete integration over phase space and summation over all polarizations leads to the $\sigma \mathrm{PF}$, but if partial integration over phase space is performed, large deviations from the $\sigma \mathrm{PF}$ are obtained. A comment on a possible improvement of the theoretical results for LFV decay rates, including their dependence on the production mechanism, is given.

Acknowledgements The author is grateful to the Workshop organizers and particularly to A. Seiden for the opportunity to attend the Workshop and to present this talk. Author is indebted to S. Eidelman for suggesting the study the problem presented here, and also thanks Z. Was, S. Eidelman, A. Czarnecki, K. Inami, A. Stahl and M. Hayashii for very instructive discussions.

Note After the author sent this paper to arXiv.org, Y. Okada draw his attention to the paper [7], in which spin correlations in the leptonic LFV tau-lepton decays were discussed in detail.

\section{REFERENCES}

1. J.D. Jackson, Nuovo Cimento 34 (1964) 1544.

2. J. Pišút, M.Ross, Nucl. Phys. B 6 (1968) 325.

3. H.M. Pilkuhn, Relativistic Particle Physics, Springer-Verlag New York Inc., 1979.

4. P. Lichard, Acta Phys. Slov 49 (1999) 215.

5. E. Byckling and K. Kajantie, Particle Kine- matics, John Wiley \& Sons, London, 1973.

6. R.N. Mohapatra and J.W.F. Valle, Phys. Rev. D 34 (1986) 1642; Bernabeu et al. Phys. Lett B 187 (1987) 303; A. Pilaftsis, Z. Phys. C 55, 275 (1992), A. Ilakovac and A. Pilaftsis, Nucl. Phys. B 437 (1995) 491; A. Ilakovac, Phys. Rev. D 62 (2000) 36010.

7. R. Kitano and Y. Okada, Phys. Rev. D 63 (2001) 113003. 